Щ्)FRANÇAISE

$\gg \mathrm{DE}$

필 PEAGOGIE

\section{Revue française de pédagogie}

Recherches en éducation

155 | avril-juin 2006

La motivation scolaire : approches récentes et perspectives pratiques

\title{
Buts de performance et de maîtrise et interactions sociales entre étudiants : la situation particulière du désaccord avec autrui
}

Performance and mastery goals and social interactions between students: the specific situation of disagreeing with others

Objetivos de performancia y de dominio e interacciones sociales entre estudiantes: la situación particular del desacuerdo con la persona ajena Leistungs- und Beherrschungsziele und soziale Interaktionen unter Studenten: der besondere Stellenwert der Meinungsverschiedenheit mit anderen

Céline Darnon, Céline Buchs et Fabrizio Butera

\section{OpenEdition Journals}

\section{Édition électronique}

URL : http://journals.openedition.org/rfp/84

DOI : $10.4000 /$ rfp. 84

ISSN : 2105-2913

Éditeur

ENS Éditions

Édition imprimée

Date de publication : 1 juin 2006

Pagination : 35-44

ISBN : 978-2-7342-1047-4

ISSN : 0556-7807

Référence électronique

Céline Darnon, Céline Buchs et Fabrizio Butera, «Buts de performance et de maîtrise et interactions sociales entre étudiants : la situation particulière du désaccord avec autrui », Revue française de pédagogie [En ligne], 155 | avril-juin 2006, mis en ligne le 20 septembre 2010, consulté le 10 décembre 2020. URL : http://journals.openedition.org/rfp/84 ; DOI : https://doi.org/10.4000/rfp.84 


\title{
Buts de performance et de maîtrise et interactions sociales entre étudiants : la situation particulière du désaccord avec autrui*
}

\author{
Céline Darnon, Céline Buchs, Fabrizio Butera
}

D'après de nombreuses recherches (voir Dweck, 1986 ; Nicholls, 1984), les étudiants peuvent poursuivre deux types de buts lorsqu'ils réalisent une tâche d'apprentissage: le but de maîtrise (désir d'apprendre, de développer des connaissances) et le but de performance (désir de mettre en avant ses compétences, ses capacités). Cet article présente une synthèse de travaux portant sur les effets des buts dans la situation particulière où l'étudiant est amené à échanger avec un autre étudiant dont la réponse diverge (situation dite de " conflit sociocognitif ", Doise \& Mugny, 1997). D’une manière générale, les résultats des études présentées indiquent que le but de maîtrise favorise la régulation épistémique (régulation du conflit centrée sur la tâche, la compréhension du problème). De plus, il rend le conflit bénéfique à l'apprentissage. Le but de performance favorise en revanche la régulation relationnelle (régulation centrée sur l'affirmation de ses compétences) et rend le conflit néfaste à l'apprentissage.

Descripteurs (TEE) : conditions d'apprentissage, conflit, dissonance cognitive, étudiant, performance, relations interpersonnelles.

\section{L'APPROCHE DES BUTS D'ACCOMPLISSEMENT}

Lorsqu'à l'université les étudiants doivent travailler sur une tâche d'apprentissage, par exemple étudier un texte, ceux-ci peuvent poursuivre une multitude de buts. Se faire des amis, vouloir faire plaisir à ses parents, éviter de travailler en sont des exemples.
Néanmoins, parmi l'ensemble des buts présents lors de la réalisation d'une telle tâche, celui d'être compétent est l'un des principaux (Nicholls, 1984). Les tâches d'apprentissage sont en effet des tâches que certains auteurs qualifient de "tâches d'aptitudes ". L'une des caractéristiques de ce type de tâches est qu'il y existe une solution plus correcte

\footnotetext{
* Ce travail a été soutenu par le programme «École et Sciences cognitives " du ministère français de la Recherche et par le Fonds national suisse de la recherche scientifique.
} 
que les autres. Ainsi, donner une réponse ou une autre permet d'assigner aux individus une valeur sociale en termes de compétences (Butera \& Mugny, 2001 ; Mugny et al., 2003). C'est pourquoi lorsqu'ils poursuivent une tâche d'aptitudes, les individus sont fortement motivés par l'atteinte de la compétence. Or, les buts répondant à cette recherche de compétence sont qualifiés de "buts d'accomplissement " (achievement goals).

Mais que signifje être compétent dans une situation académique? Prenons l'exemple d'un étudiant fraîchement arrivé à l'université. Réussir ses études constitue un enjeu majeur pour ce dernier. Mais que signifie pour lui "réussir"? Deux cas de figure peuvent être distingués. II se peut en effet que " réussir " signifie pour cet étudiant « apprendre des choses " c'est-à-dire augmenter son niveau de connaissance et plus généralement de compétence. Supposons que la fin de l'année approche et qu'il cherche à estimer la manière dont il a atteint son but. Comment va-t-il le faire? II est probable que, si pour l'étudiant en question, « réussir » signifie « progresser », celui-ci va opérer une comparaison intraindividuelle, c'est-à-dire qu'il évaluera l'état de ses connaissances à la fin de l'année et le comparera à l'état des connaissances qu'il avait en arrivant à l'université. Cependant, un autre cas de figure est possible. Il se peut en effet que pour cet étudiant, "réussir" signifie "être reconnu compétent". Comment l'individu va-t-il faire, en fin d'année pour évaluer sa réussite, c'est-à-dire l'atteinte de son but d'accomplissement? La solution la plus informative, dans ce cas, est de comparer sa réussite à celle des autres étudiants. La comparaison est alors interindividuelle et normative, c'est à dire socialement référée.

Pour de nombreux auteurs (voir par exemple Nicholls, 1984), ces deux manières de définir la compétence correspondent à deux types de buts d'accomplissement. Le premier est qualifié de but d'implication dans la tâche ou but de maîtrise. Le second est appelé but d'implication dans l'ego ou but de performance (Ames, 1992 ; Dweck, 1986). Le but de maîtrise implique une évaluation des compétences propres basée sur l'amélioration de la maîtrise de la tâche, il correspond donc au désir d'apprendre, de comprendre le problème, d'acquérir de nouvelles connaissances. Le but de performance en revanche repose sur une définition de ses compétences en rapport avec celles des autres, il correspond donc au désir de se montrer meilleur que les autres. Pour les auteurs, ces buts peuvent à la fois relever de dispositions personnelles (certains étudiants poursuivraient principalement un but de maîtrise, d'autres un but de performance) et de facteurs situationnels (il existe des situations favorisant l'adoption du but de maîtrise, d'autres favorisant l'adoption de but de performance).

De nombreuses études indiquent que l'adoption de l'un ou l'autre de ces buts affecte la réaction des individus face à la tâche d'apprentissage, tels que les attributions qu'ils font en cas d'échec, la représentation qu'ils ont de l'effort, ainsi que les stratégies qu'ils utilisent pour étudier (pour une revue, voir Cosnefroy, 2004 ; Darnon \& Butera, 2005). Dans l'ensemble, Dweck (1986) qualifie les comportements découlant du but de maîtrise " d'adaptatifs" (adaptive) et ceux découlant du but de performance de "non-adaptatifs" (maladaptive).

La littérature portant sur l'impact des buts de maîtrise et de performance est abondante et très fournie. II est surprenant néanmoins de constater que la majorité des études menées dans ce domaine s'intéresse aux réactions des élèves et des étudiants seulement sur un plan intra-individuel. Pourtant la plupart des tâches d'apprentissage sont réalisées au sein de classes, à l'école comme à l'université, c'est-à-dire dans des contextes incluant d'autres personnes, avec qui l'étudiant peut avoir à échanger et à discuter.

Or quelles peuvent ëtre les implications de la prèsence d'autrui ? Comme noté par Dweck et Leggett (1988), lorsqu'ils poursuivent un but de maîtrise, les étudiants sont intéressés par la question «Comment faire pour augmenter mes capacités, pour réussir à maîtriser la tâche? " Lorsqu'ils poursuivent un but de performance en revanche, les étudiants sont intéressés par la question : "est-ce que je suis compétent ? " La présence d'une autre personne permet d'apporter une réponse à ces deux questions à la fois. En effet, autrui peut représenter à la fois un support informationnel et une source de comparaison sociale (Buchs, Butera \& Mugny, 2004). Lorsque celui-ci essaie de résoudre le même problème, il est susceptible de proposer d'autres solutions, d'autres explications, d'autres exemples. D'un coté, ces informations peuvent servir de support informationnel, dans la mesure où elles peuvent aider l'individu à résoudre le problème et de fait, à maîtriser la tâche (voir par exemple Newman, 1990). D'un autre, les réponses données par cet autrui donnent également des informations sur sa compétence, et donc également, par comparaison sociale, sur sa propre compétence (Butera \& Muller, 2004). 


\section{UNE SITUATION SOCIALE PARTICULIÈRE : LE CONFLIT SOCIOCOGNITIF}

\section{Effets des buts en fonction du conflit}

Il apparaît donc qu'autrui, puisqu'il est un élément qui est susceptible d'apporter à la fois des informations sur la tâche, et sur la compétence, présente la particularité de renforcer à la fois les enjeux de maîtrise et ceux de performance. Or il existe une situation où ceci devrait être particulièrement le cas : lorsque la réponse que donne cet autrui diverge de la réponse propre. En effet, être confronté à un autrui en désaccord souligne le fait que la tâche n'est peut-être pas maîtrisée (" s'il existe une autre solution possible, peut-être cela signifie-t-il que la solution que j'ai proposée n'est pas correcte ? "), et remet en même temps en question la compétence propre («peut être l'autre personne est-elle plus compétente que moi ? ", Butera \& Mugny, 1995; Quiamzade \& Mugny, 2001). Le conflit rend donc les individus doublement incertains.

Or il apparaît de la littérature sur les buts que l'incertitude, et plus exactement la mesure dans laquelle la tâche peut entraîner de la peur de l'échec, est justement un élément extrêmement important pour expliquer les effets des buts. En effet, il ressort des différentes études s'intéressant au lien entre buts et apprentissage que le but de maîtrise augmente l'apprentissage lorsque la tâche est "difficile" (Covington \& Omelich, 1984; Elliot \& McGregor, 1999 ; Grant \& Dweck, 2003) ou confuse (Licht \& Dweck, 1984). II apparaît également que le but de performance n'a d'effets délétères sur l'apprentissage que lorsqu'il est associé à des échecs (Dweck \& Legett, 1988; Diener \& Dweck, 1980), à une faible perception des compétences propres (Elliott \& Dweck, 1988 ; Nicholls, 1984), ou lorsque la tâche est considérée comme difficile (Grant \& Dweck, 2003).

Le conflit implique une forte incertitude et de la peur de l'échec. C'est donc dans cette situation que des différences entre but de performance et but de maîtrise devraient s'observer. On peut donc penser que les effets des buts sur l'apprentissage varient selon que la situation implique ou non, un conflit.

\section{Effets du conflit en fonction des buts}

Cette situation de confrontation de points de vue contradictoires renvoie à ce que certains auteurs appellent le « conflit sociocognitif » (Doise \& Mugny, 1997 ; voir également pour une revue de questions :
Buchs et al., 2004 ou la controverse Johnson \& Johnson, 1993; Johnson, Johnson \& Tjosvold, 2000). Or ces auteurs montrent que, outre le fait que le conflit augmente l'incertitude et la peur de l'échec, celui-ci peut également représenter un potentiel intéressant pour l'apprentissage. Les travaux réalisés en psychologie sociale du développement cognitif par exemple (Doise \& Mugny, 1997) montrent que ce type de conflit peut favoriser les progrès cognitifs. D'autres auteurs suggèrent également que le conflit peut favoriser l'apprentissage (Lowry \& Johnson, 1981), la curiosité épistémique et la qualité du raisonnement (Smith, Johnson \& Johnson, 1981). Enfin, les recherches menées dans le domaine de l'influence sociale montrent également le rôle du conflit dans la transmission de connaissances (Butera et al., 1998 ; Mugny et al., 2003).

Néanmoins, tous soulignent que si le conflit peut faire progresser et apprendre, ce n'est pas toujours le cas. En effet, dès lors qu'il s'associe à une menace pour les compétences (Buchs \& Butera, 2004 ; Mugny et al., 2003) ou à de la compétition (Johnson \& Johnson, 1985 ; Butera \& Mugny, 1995), le conflit perd ses bénéfices. C'est ce qui amène les auteurs à considérer que le conflit peut être régulé de deux manières différentes. En effet, lorsque la régulation du conflit est centrée sur la tâche, la compréhension du problème, alors elle est dite "épistémique ». Si en revanche, pour réguler le conflit, les individus se centrent sur la comparaison sociale des compétences avec leur partenaire, alors la régulation du conflit est dite «relationnelle" (Butera \& Mugny, 2001; Mugny, De Paolis \& Carugati, 1984). Le conflit n'a d'effets positifs que lorsqu'il est régulé de manière épistémique.

Nous avons vu par ailleurs qu'il était probable que le but de maîtrise amène à percevoir l'autre comme source de support informationnel alors que le but de performance devrait amener à le percevoir comme une menace pour les compétences. On peut donc s'attendre à ce que le conflit, lorsqu'il s'associe à un but de maîtrise, favorise la régulation épistémique et l'apprentissage. En revanche, le conflit, associé à un but de performance devrait favoriser la régulation relationnelle et perdre ses bénéfices.

\section{CONFLIT ET BUTS : UNE MODULATION RÉCIPROQUE}

Cet article vise à présenter une synthèse de recherches portant sur les effets interactifs des buts 
et du conflit. Plus spécifiquement, nous rapporterons les principaux résultats d'études testant l'hypothèse selon laquelle les effets du confit peuvent dépendre des buts que poursuivent les étudiants lorsqu'ils réalisent la tâche, ainsi que l'hypothèse complémentaire selon laquelle les effets des buts dépendent de la conflictualité de l'interaction.

Nous avons déjà évoqué le fait que les buts pouvaient relever de variable de personnalité, comme de facteurs plus situationnels. Par conséquent, deux méthodologies peuvent être employées pour étudier les buts. Dans certaines études en effet, les buts sont manipulés (par exemple, la tâche est présentée soit comme un moyen d'apprendre des choses, soit comme un moyen d'évaluer les compétences). Dans d'autres études, les buts sont mesurés comme variables dispositionnelles, c'est à dire que chaque participant doit indiquer la mesure dans laquelle il poursuit un but de maîtrise ou de performance.

Nous allons rapporter dans un premier temps deux études réalisées en laboratoire, où les buts ont été manipulés. Pour cela, des consignes mettant en avant la nécessité d'apprendre (but de maîtrise) ou d'être performant, de faire preuve de compétence (but de performance) ont été élaborées. Une étude pilote a permis d'attester que ces consignes affectaient la manière dont les étudiants répondaient sur une échelle de mesure des buts d'accomplissement. Les deux études que nous présenterons ensuite ont été réalisées au sein de classes et les buts y ont été mesurés comme variables dispositionnelles. Pour cela, une échelle de mesure des buts d'accomplissement, celle d'Elliot et McGregor (2001) a été traduite et validée (Darnon \& Butera, 2005).

\section{Effets interactifs des buts et du conflit sur le mode de régulation du conflit et l'apprentissage}

Dans une première étude (Darnon, 2004 ; Darnon, Butera \& Harackiewicz, 2006), nous explorions les effets interactifs des buts et du conflit sur l'apprentissage. Celle-ci était présentée comme une étude d'apprentissage coopératif médiatisé par ordinateur. Le paradigme utilisé ici consistait à faire croire aux participants qu'ils interagissaient avec un autre des participants par l'intermédiaire d'un ordinateur, à propos d'un texte à étudier. Ce texte présentait des effets liés au témoignage oculaire et était issu d'un ouvrage de psychologie sociale.

Les étudiants (des étudiants de psychologie) étaient accueillis par groupes de quatre au labora- toire et recevaient, en fonction de la condition expérimentale, des consignes de maîtrise (par exemple: "Il s'agit pour vous d'acquérir de nouvelles connaissances qui pourront vous être utiles par la suite, de bien comprendre les expériences, les exemples et les idées du texte, de découvrir de nouveaux concepts ") ou des consignes de performance (par exemple: "II s'agit pour vous d'être performant, d'être bon, donc d'avoir une bonne note au QCM final "). Dans un groupe contrôle, ils ne recevaient pas de consigne particulière. Après avoir reçu ou non ces consignes, ils étaient tous sensibilisés à l'importance de prendre en compte le point de vue de leur partenaire (et ce grâce à une tâche de décentration, voir Huguet, Mugny \& Pérez, 1991-1992 pour un détail de cette tâche). Ils étaient ensuite séparés dans différents boxes expérimentaux, chacun face à un ordinateur.

La tâche, sur ordinateur consistait à lire une partie du texte à étudier, puis à répondre à une question sur ce texte. Les participants devaient alors " envoyer " leur réponse à leur soi-disant partenaire. Après un temps d'attente, ils recevaient une "réponse ", supposée venir de leur partenaire. En réalité, il s'agissait d'une réponse préprogrammée de manière à introduire ou non un conflit. La réponse que donnaient les participants était prévisible. De fait, la réponse préprogrammée avait été conçue de manière à être en accord ou en désaccord avec celle des participants. Dans la condition de conflit, le supposé « partenaire » était en désaccord (par exemple : "il me semblait plutôt que..."). Dans la condition sans conflit, il était en accord (par exemple: «ok, j'aurais répondu la même chose ").

Cette procédure était menée quatre fois. Puis les participants devaient répondre à quelques questions sur leur incertitude, la mesure dans laquelle la réponse de l'autre avait introduit de la peur de l'échec. Enfin, ils répondaient à un questionnaire à choix multiple de compréhension du texte, celui-ci servant de mesure d'apprentissage.

Les résultats de cette étude montrent, d'une manière générale, que le conflit favorise l'incertitude et la peur de l'échec. Ceci apparaît notamment grâce à un effet principal du conflit sur la perception des compétences propres et sur la peur de l'échec. II favorise également ce que nous qualifions de "tentatives pour réduire cette incertitude", telles que les retours au texte et le choix de répondre au partenaire. Plus important pour notre propos, les résultats indiquent que comme attendu, les effets du 
conflit dépendent des conditions de buts. Dans la condition de but de maîtrise, les désaccords entraînent un meilleur apprentissage que les accords. L'inverse se produit dans la condition de but de performance où les désaccords détériorent l'apprentissage, comparativement à la condition d'accord. Cette même interaction indique également que la condition de but de maîtrise n'entraîne un meilleur apprentissage que celle de but de performance que lorsqu'il y a un conflit. Dans les conditions d'accord, ces conditions de buts sont équivalentes.

Rappelons que dans le groupe contrôle, aucune consigne particulière n'était donnée. Dans cette condition, la différence entre les conditions d'accord et désaccord n'atteint pas le seuil de significativité. Néanmoins, il est intéressant de noter que les résultats obtenus dans cette condition sont particulièrement similaires aux résultats obtenus dans la condition de but de performance. II semblerait donc que par défaut, le but le plus prégnant dans le groupe contrôle soit un but de performance.

Ces résultats indiquent donc que comme attendu, c'est lorsqu'il y a un conflit que les buts ont des effets différentiels sur l'apprentissage. Ils montrent en outre que les effets du conflit dépendent fortement du but souligné par le contexte. En effet, dans un contexte mettant en avant le but de maîtrise, le conflit a un effet positif. Dans un contexte prônant le but de performance en revanche, il a un effet négatif. Comme nous l'avons souligné plus haut, il est possible que cet effet soit dû au mode de régulation du conflit. Dans une seconde étude, nous avons donc examiné les effets interactifs des buts et du conflit, cette fois, sur son mode de régulation.

Dans cette étude (Darnon, 2004 ; Darnon \& Butera, 2006), les participants, également des étudiants de psychologie, discutaient réellement entre eux, à propos de textes présentant des théories contradictoires (par exemple, le texte A présentait l'effet de facilitation sociale, le texte $B$ l'effet d'inhibition sociale). Le conflit n'était donc pas manipulé, mais mesuré. Pour cela, les participants devaient indiquer, à la fin de l'échange, quelle était la quantité d'éléments sur lesquels ils avaient été en désaccord avec leur partenaire. Ils devaient également indiquer dans quelle mesure, lorsque ce désaccord s'était produit, ils l'avaient régulé de manière épistémique (par exemple, "avez-vous essayé de voir en quoi chacune des propositions pouvaient vous aider à comprendre") ou relationnelle (par exemple, "avezvous essayé de montrer que vous aviez raison »).
Comme dans l'étude précédente, les étudiants avaient reçu, avant l'échange, des consignes soit de performance, soit de maîtrise, soit pas de consignes particulières.

Les résultats de cette étude indiquent, comme attendu, que le désaccord perçu prédit un mode de régulation different en fonction de la condition de buts. En effet, le désaccord prédit la régulation épistémique du conflit, uniquement dans la condition de maîtrise. Ce n'est pas le cas dans les deux autres conditions. De plus, le désaccord ne prédit la régulation relationnelle du conflit que sous consigne de performance. Ce n'est pas le cas dans les deux autres conditions.

Ces études indiquent donc qu'il est possible d'orienter les buts poursuivis par les étudiants en leur donnant des consignes de travail particulières. Des études complémentaires soulignent que la distribution des informations (Buchs, à paraître; Buchs \& Butera, 2004 ; Buchs et al., 2004 ; Darnon, Buchs \& Butera, 2002) pourrait contribuer à orienter les buts lors d'un travail de groupe. Ainsi lorsque les étudiants travaillent sur des informations identiques, ils sont plus fortement orientés vers un but de performance. Dans ce climat plus compétitif, la compétence du partenaire est menaçante et entrave l'apprentissage des étudiants; les conflits ne sont pas bénéfiques à l'apprentissage. Au contraire, quand les étudiants travaillent sur des informations complémentaires, le but de performance est moins fort et les interactions sociales sont plus positives. Le partenaire est perçu comme un support informationnel, sa compétence est alors reconnue et bénéfique pour l'apprentissage des étudiants. C'est dans ce climat plus coopératif que les conflits sociocognitifs sont le plus positivement liés à l'apprentissage.

\section{Buts mesurés comme variables dispositionnelles et régulation du conflit}

La question à approfondir maintenant est de savoir ce qu'il en est lorsque les buts sont envisagés non pas comme relevant du contexte, mais comme relevant de prédispositions particulières. On peut penser là aussi, que les individus poursuivant un but de maîtrise ne perçoivent pas le partenaire, et en particulier le partenaire en conflit, de la même manière que lorsqu'ils poursuivent plutôt un but de performance. En d'autres termes, poursuivre un but de maîtrise devrait amener à percevoir l'autre comme une source d'information, ce qui en cas de conflit, devrait favoriser la régulation épistémique. Le but de 
performance en revanche devrait amener à percevoir l'autre comme une menace. Ainsi, le but de performance devrait amener, en cas de conflit, à défendre de son propre point de vue, en dépit de celui de l'autre, de manière à protéger la compétence propre et à invalider celle de l'autre. Le but de performance devrait donc favoriser la régulation relationnelle. C'est ce que nous avons testé au sein des deux études que nous allons présenter ici (Darnon, 2004) (1).

La troisième étude a été réalisée lors d'une séance de travaux dirigés (TD) de psychologie sociale, sur un public d'étudiants de deuxième année de psychologie. Dans ces TD, une méthode d'apprentissage coopératif (inspirée des "classes puzzle" - « Jigsaw classrooms "- Aronson \& Patnoe, 1997 ; voir Buchs, Filisetti et al., 2004) était instituée. Durant le TD en effet, les étudiants étaient amenés, à deux reprises, à interagir entre eux. Le premier temps d'interaction se faisait entre personnes ayant travaillé sur une même thématique (groupe d'experts). Dans un deuxième temps, d'autres groupes étajent formés et étaient composés de trois étudiants ayant travaillé sur des thématiques différentes. Durant cette phase, chacun devait exposer sa thématique à ses deux "partenaires ", et écouter chacun d'entre eux exposer la leur. II leur était vivement conseillé d'interagir, de poser des questions et d'être actifs pendant l'ensemble de ces phases. Ces temps d'échanges permettaient de rendre pertinentes les questions sur le conflit et sur la régulation du conflit.

Ainsi, les étudiants devaient répondre à un questionnaire de mesure des buts, puis rapporter la quantité d'éléments sur lesquels ils s'étaient trouvés en désaccord avec leurs pairs, durant les temps de discussion qu'ils avaient eus en classe. Ils devaient ensuite rapporter dans quelle mesure, lorsqu'il y avait eu désaccord, ils avaient régulé celui-ci de manière relationnelle ou épistémique.

Les résultats de cette étude indiquent que l'adoption du but de maîtrise favorise la régulation épistémique. L'adoption du but de performance prédit pour sa part la régulation relationnelle.

Ces résultats confirment donc les attentes. Néanmoins, il est important de noter que les questions sur le mode de régulation du conflit portent sur les désaccords durant les temps d'interactions du TD. Or les moyennes indiquent que la quantité de désaccord durant ces interactions est relativement peu élevée (la moyenne est de 2,5 sur une échelle allant de 1 à 7). Répliquer ces résultats au sein d'un contexte où les désaccords sont assurés par la confrontation à un autrui, dont les réponses seraient standardisées, nous a donc paru important.

Une quatrième étude a donc visé à répliquer ces résultats dans une situation similaire aux conditions de conflit de la première étude à savoir la simulation d'interaction sociale médiatisée par ordinateur. Les participants (des étudiants de psychologie) étaient amenés à croire qu'ils interagissaient avec un partenaire, lequel était toujours en conflit. Ceci permettait de s'assurer que la quantité de conflit serait importante. Le questionnaire des buts d'accomplissement ainsi que les items de régulation du conflit étaient les mêmes que ceux utilisés pour l'étude précédente.

Les résultats de cette étude permettent de répliquer intégralement les résultats observés dans l'étude précédente. En effet, le but de maîtrise prédit la régulation épistémique, le but de performance la régulation relationnelle.

\section{Synthèse des résultats et perspectives}

Dans leur ensemble, les résultats des quatre études dont la synthèse est présentée ici soutiennent l'hypothèse générale. Qu'il s'agisse d'un but mis en avant par une consigne particulière ou d'un but adopté par les individus pour des raisons qui leurs sont propres, le but de maîtrise crée un contexte dans lequel le conflit peut être régulé de manière épistémique et peut s'avérer bénéfique à l'apprentissage. Le but de performance en revanche crée un contexte dans lequel le conflit menace les compétences propres, est régulé de manière relationnelle et devient néfaste à l'apprentissage. Comme mentionné plus haut, dans ces études, les buts sont à la fois considérés comme variables dispositionnelles (et mesurés comme tels), et comme variables dépendantes du contexte (et donc, manipulables). II est intéressant de noter que les résultats obtenus avec ces deux approches sont tout à fait cohérents, soulignant que quelle que soit l'origine de l'adoption de certains buts ou d'autres, ceux-ci s'avèrent avoir des effets importants sur la manière dont le conflit est régulé et sur ses effets sur l'apprentissage.

Notons que les études présentées ici ont été réalisées sur des étudiant(e) s en psychologie. Retrouverait-on des dynamiques similaires sur d'autres populations, telles que, par exemple, des étudiants d'autres filières, ou des populations plus jeunes? 
Certains éléments de la littérature indiquent que les buts ainsi que leurs effets sur différentes variables, évoluent avec l'age (Anderman, Austin \& Johnson, 2002). Par ailleurs les chercheurs dans le domaine du conflit (Doise \& Mugny, 1997) indiquent que les modes de régulation sont rendus possible par certaines capacités cognitives, lesquelles évoluent aussi avec l'âge. L'examen de nos hypothèses sur d'autres populations, notamment sur des enfants, pourra en ce sens faire l'objet de recherches futures et contribuer à la compréhension des mécanismes de régulation du conflit.

Il est important de souligner également que la régulation du conflit est une variable éminemment sociale. En effet, réguler le conflit de manière épistémique, c'est reconnaître ce en quoi la réponse donnée par autrui peut aider à accéder à une meilleure compréhension du problème. Réguler le conflit de manière relationnelle, c'est en revanche dénigrer la validité de la réponse de l'autre et affirmer la compétence propre. Une piste à explorer pourrait être en ce sens l'examen de la nature des affinités existantes entre participants du conflit comme variable modératrice des effets des buts d'une part et du conflit de l'autre. En particulier, deux hypothèses pourraient être émises à ce sujet. On peut penser que face à un conflit, deux individus entretenant une relation très positive soient très soucieux du respect d'autrui et de ses idées. Ceci favoriserait sans doute la régulation épistémique quel que soit le but adopté. On peut néanmoins penser qu'une relation très positive avec autrui rende saillante la nécessité d'éviter le conflit, en vue de préserver la relation. Dans ce cas, il est possible de penser qu'une relation très positive favorise la complaisance, une forme de régulation relationnelle du conflit. Dans la lignée des recherches présentées ici, un examen de ces hypothèses contribuerait grandement à l'intégration de la question du « social » dans l'étude des buts d'accomplissement.

\section{IMPLICATIONS PRATIQUES}

Comme attendu, cet ensemble de recherche souligne que la situation d'apprentissage peut prendre un sens différent en fonction de 1) les buts que poursuivent les étudiants (et ce, que ces buts relèvent de traits dispositionnels ou de la situation) et 2) de la nature de l'interaction avec les autres, ici, la conflictualité de l'échange. Plus exactement, ils montrent que le but de maîtrise favorise la régulation épistémique, et rend le conflit bénéfique à l'apprentissage. Le but de performance favorise en revanche la régulation relationnelle, et rend le conflit néfaste à l'apprentissage.

Même si des recherches supplémentaires sont nécessaires pour confirmer et compléter ces résultats, ceux-ci contribuent déjà grandement à la compréhension des dynamiques présentes à l'université et de leur potentiel tantôt positif tantôt négatif pour l'apprentissage. Les deux éléments que nous avons examinés ici, conflit d'une part, but de maîtrise ou de performance d'autre part sont d'autant plus important à étudier qu'ils font l'objet d'un certain nombre de croyances qui peuvent interférer avec leur usage constructif.

Par exemple, il existe une croyance générale selon laquelle créer de la compétition interpersonnelle est un bon moyen de " motiver " les étudiants et de les impliquer dans leurs activités. Ceci explique sans doute que la compétition soit si présente dans les systèmes éducatifs (voir, pour une discussion de ce point, Butera \& Buchs, 2004 ; Monteil, 1989). Ceci est particulièrement le cas à l'université, où l'étudiant ne cesse d'entendre, dès son entrée en première année, qu'étant donné le taux d'échec, réussir signifiera pour lui faire partie des $38 \%$ des meilleurs (2). Or, ceci ne fera que s'accentuer avec les années d'études, où en même temps que le nombre de personnes admises diminue, on ne cesse de rappeler à l'étudiant que pour faire sa place (par exemple être accepté en troisième cycle), il ne suffit pas de réussir, mais il faut réussir mieux que les autres.

Une seconde croyance, encore assez répandue dans les systèmes éducatifs, est qu'il est parfois préférable que les élèves (plus tard, les étudiants) ne soient pas en conflit les uns avec les autres. Même si les formations d'enseignants sensibilisent maintenant ces derniers à l'intérêt du conflit en classe, il semble que celui-ci reste particulièrement difficile et délicat à mettre en place. En effet, comme le notent Pérez et al. (1995), le conflit est culturellement vu comme "mauvais". De plus, du fait que les individus ont l'impression qu'il implique nécessairement des tensions relationnelles, il serait perçu comme particulièrement risqué. Pour Johnson, Johnson et Smith (2000), il existe deux raisons principales pour lesquelles les conflits auraient tendance à être évités en classes : d'une part, pour qu'il y ait conflit, il faut que les étudiants puissent discuter entre eux. Or, 
selon eux, la plupart des classes sont structurées de manière à éviter les discussions entre étudiants. La seconde raison est que les individus pensent que pour pouvoir être "critique " (et donc participer au conflit), il faut disposer d'abord d'une base de connaissances solide et correcte. Ceci amène à penser que seuls les experts pourraient être en conflit les uns avec les autres. De fait, les conflits sont la plupart du temps évités dans les classes. Or, là encore, il semblerait que ceci soit particulièrement le cas à l'université. La structure même des cours magistraux en amphithéâtre accentue l'asymétrie de statut entre le professeur et les étudiants, et rappelle les rôles bien définis entre "celui qui sait " (l'enseignant) et «ceux qui apprennent » (les étudiants). De plus, et également du fait de leur taille, les amphithéâtres rendent particulièrement difficile la gestion des échanges entre étudiants. De fait, même si parfois, lors de leurs travaux dirigés, les étudiants peuvent être amenés à interagir entre eux, cette pratique reste relativement limitée. Les étudiants ayant peu la possibilité d'échanger, ils ont donc particulièrement peu la possibilité d'échanger sur des points de vue contradictoires.

La revue de la littérature amène à penser que ces deux croyances sont regrettables. En effet, la compétition, et avec la compétition, le but de performance, a bien souvent des effets délétères sur la manière dont la tâche est traitée. D'autre part, le conflit peut favoriser l'apprentissage (Buchs et al., 2004). Les travaux que nous avons présentés ici indiquent néanmoins qu'il ne convient ni de diaboliser la norme compétitive, ni de prôner à tout prix les conflits. Bien plus, ils montrent comment ces deux éléments peuvent agir et interagir de manière à parfois favoriser l'apprentissage, d'autres fois le détériorer.

Les résultats de ces études indiquent par exemple qu'associé à un but de performance, le conflit a un effet négatif. La similarité entre groupe contrôle et groupe de performance indique par ailleurs que par défaut, le but qui semble le plus présent à l'université est un but de performance. On pourrait déduire de ce résultat, que puisque le but de performance domine au sein du système éducatif, et que, associé à un but de performance, le conflit est régulé de manière relationnelle ce qui entraîne un moins bon apprentissage que l'absence de conflit, alors il est préférable d'éviter le conflit en classe. Ce point de vue serait cohérent avec la vision négative du conflit que nous avons évoquée plus haut. II serait également cohérent avec certaines méthodes pédagogiques actuelles consistant à éviter autant que faire se peut les feed-back négatifs, les indications d'échecs, tout ce qui signifie à l'élève ou l'étudiant qu'il peut se tromper, ou s'être trompé. II présenterait en outre l'avantage de la facilité puisque comme le suggèrent Johnson, Johnson et Tjosvold (2000), il est beaucoup plus facile pour l'enseignant de faire un cours de façon magistrale et ainsi de garder le contrôle sur ce que font les étudiants.

Néanmoins, nos résultats indiquent également que le but de performance n'est pas incontournable, puisqu'il est possible d'introduire un but de maîtrise. De plus, ils indiquent qu'associé à un but de maîtrise, le conflit favorise la régulation épistémique et l'apprentissage. De fait, plutôt que d'éviter le conflit, de manière à s'assurer que les étudiants n'en subiront pas d'effets délétères, ne serait-il pas judicieux d'agir sur la structure de classe, les consignes, la présentation du travail, etc. (pour une revue des différents facteurs susceptibles d'orienter vers l'un ou l'autre des buts, voir par exemple Ames, 1992; Maehr \& Midgley, 1991) de manière à ce que celuici favorise le but de maîtrise ? Dans un tel contexte, non seulement le confit n'aura pas d'effet délétère, mais il pourra en outre augmenter l'apprentissage. II sera donc tout à l'avantage de l'étudiant.

Céline Darnon celine.darnon@upmf-grenoble.fr Université Pierre Mendès-France (Grenoble 2) Laboratoire de psychologie sociale University of Wisconsin-Madison (USA)

Department of Psychology

Céline Buchs

celine.buchs@pse.unige.ch

Université de Genève

Section des sciences de l'éducation

Fabrizio Butera

fabrizio.butera@unil.ch

Université de Lausanne

Institut des sciences sociales et pédagogiques 
(1) I| est important de noter que ces deux études comprenaient également une mesure du but de performance-évitement (voir Elliot, 1997 ; Elliot \& Harackiewicz, 1996). Pour une question de simplification, nous nous limiterons ici à présenter les résultats concernant le but de maitrise et le but de performance (-approche)
(2) C'est le pourcentage d'étudiants inscrits en première année de DEUG de psychologie en 2003 à l'université Pierre MendèsFrance (Grenoble 2) qui ont réussi cette première année. Notons qu'il n'est pas rare que le pourcentage annoncé soit, dans les discours des étudiants comme dans ceux des enseignants, un pourcentage encore plus bas que ce pourcentage réel.

\section{BIBLIOGRAPHIE}

AMES C. (1992). "Classrooms: Goals, structures, and students motivation ". Journal of Educational Psychology, vol. 84 , p. 261-271.

Anderman E. M. ; Austin C. C. \& Johnson D. M. (2002). "The development of goal orientation ». In A. Wigfield \& J. Eccles (éd.), Developmental perspectives on achievement motivation. San Diego: Academic Press, p. 197220.

ARONson E. \& PATNoe S. (1997). Cooperation in the classroom: The jigsaw method. New York : Longman.

Buchs C. (à paraître). "La distribution des informations dans les dispositifs d'apprentissage entre pairs ». In Y. Rouiller \& K. Lehraus (éd.), L'apport des approches coopératives pour les apprentissages.

Buchs C. \& BUTERA F. (2004). « Socio-cognitive conflict and the role of student interaction in learning $"$. New Review of Social Psychology, vol. 3, p. 80-87.

Buchs C. ; Butera F. \& Mugny G. (2004). «Resource interdependence, student interactions and performance in cooperative learning ". Educational Psychology, vol. 24, p. 291-314.

Buglis C. ; Filigetti L. ; Butcma F. \& Quiamzade ^. (2004). "Comment l'enseignant peut-il organiser le travail de groupe de ses élèves ? ". In E. Gentaz \& P. Dessus (éd.), comprendre les apprentissages : sciences cognitives et éducation. Paris : Dunod, p. 168-183.

ButerA F. \& Buchs C. (2004). " Autorité et apprentissage : des objectifs mutuellement exclusifs ? "In M.-C. Toczek-Capelle \& D. Martinot (éd.), Le défi éducatif. Paris : A. Colin, p. 227-239.

Butera F. \& Mugny G. (1995). "Conflict between incompetences and influence of a low expertise source in hypothesis testing 》. European Journal of Social Psychology, vol. 25 , p. $457-462$.

Butera F. \& MugnY G. (2001). "Conflicts and social influences in hypothesis testing ". In C. K. W. De Dreu \& N. K. De Vries (éd.), Group consensus and minority influence. Implications for innovation. London : Blackwell, p. 161-182.

Butera F. \& MulleR D. (2004). « Le besoin de se comparer ». Pour la science, $\mathrm{n}^{\circ} 315$, p. $78-81$

Butera F. ; Gardair E. ; Maggi J. \& Mugny G. (1998). « Les paradoxes de l'expertise: influence sociale et (in) compétence de soi et d'autrui ". In J. Py, A. Somat \& J. Baillé (éd.), Psychologie sociale et formation professionnelle: propositions et regards critiques. Rennes: Presses universitaires de Rennes, p. 111-123.

COSNEFROY L. (2004). "Apprendre, faire mieux que les autres, éviter l'échec: I'influence de l'orientation des buts sur les apprentissages scolaires ". Revue française de pédagogie, $\mathrm{n}^{\circ} 147$, p. 107-128.

Covington M. V. \& OMELICH C. L. (1984). "Task-oriented versus competitive learning structures: Motivational and performance consequences ". Journal of Educational Psychology, vol. 76, p. 1038-1050.

DARNON C. (2004). Conflit sociocognitif et buts d'accomplissement : effets interactifs sur l'apprentissage et le mode de régulation du conflit. Thèse de doctorat : psychologie sociale expérimentale, université Pierre MendèsFrance - Grenoble 2.

Darnon C. ; Buchs C. \& Butera F. (2002). "Epistemic and relational conflicts in sharing identical vs. Complementary information during cooperative learning ". Swiss Journal of Psychology, vol. 61, p. 139-151.

DARNON C. \& BUTERA F. (2005). «Buts d'accomplissement, stratégies d'étude, et motivation intrinsèque : présentation d'un domaine de recherche et validation française de l'échelle d'Elliot et McGregor (2001)". L'Année psychologique, vol. 105, p. 105-131.

Darnon C. ; Butera F. \& Harackiewicz J.-M. (2006). « Achievement goals in social interactions: learning with a mastery versus performance context ». [Article soumis pour publication]

DARNON C. \& BUTERA F. (2006). "Learning or Succeeding? Conflict regulation within mastery and performance goals ». [Article soumis pour publication].

DIENER C. I. \& DWECK C. S. (1980). «An analysis of learned helplessness II: The processing of success $\%$. Journal of Personality and Social Psychology, vol. 39, p. 940952.

DoIsE W. \& Mugny G. (1984). The Social Development of the Intellect. Oxford [USA] : Pergamon Press.

Doise W. \& Mugny G. (1997). Psychologie sociale et développement cognitif. Paris : A. Colin [2 $2^{\mathrm{e}}$ éd.].

DwECk C. S. (1986). "Motivational processes affecting learning ". American Psychologist, vol. 41, p. 1040-1048.

DWECK C. S. \& LEGGETT E. L. (1988). "A social-cognitive approach to motivation and personality ». Psychological Review, vol. 95, p. 256-273.

El.LIOT A. J. (1997). "Integrating the "classic" and "contemporary" approaches to achievement motivation: A hierarchical model of approach and avoidance motivation ". In M. Maehr \& P. Pintrich (éd.), Advances in motivation and achievement, vol. 10, p. 143-179.

Elliot A. J. \& HaRACKIEWICZ J. M. (1996). "Approach and avoidance achievement goals and intrinsic motivation : A mediational analysis". Journal of Personality and Social Psychology, vol. 70, p. 461-475. 
Elliot A. J. \& McGregor H. A. (1999). «Test anxiety and the hierarchical model of approach and avoidance achievement motivation ". Journal of Personality and Social Psychology, vol. 76, p. 628-644.

Elliot A. J. \& MCGRegor H. A. (2001). «A 2*2 Achievement Goal Framework ». Journal of Personality and Social Psychology, vol. 80, p. 501-519.

ELLIOTT E. S. \& DWECK C. S. (1988). "Goals : An approach to motivation and achievement ". Journal of Personality and Social Psychology, vol. 54, p. 5-12.

GrANT H. \& DWECK C. (2003). "Clarifying achievement goals and their impact ". Journal of Personality and Social Psychology, vol. 85, p. 541-553.

Huguet P. ; Mugny G. \& Pérez J. A. (1991-1992). « Influence sociale et processus de décentration ". Bulletin de psychologie, $\mathrm{n}^{\circ} 405$, p. $155-163$.

JoHnson D. W. \& Johnson R. T. (1985). « Classroom conflict : Controversy versus debate in learning groups ". American Educational Research Journal, vol. 22, p. 237-256.

JOHNSON D. W. \& JOHNSON R. T. (1993). « Structuring academic controversy". In S. Sharan (éd.), Handbook of cooperative learning methods. Westport : Greenwood.

JOHNSON D. W. ; JOHNSON R. T. \& SMITH K. (2000). " Constructive controversy: The educative power of intellectual conflict ". Change, vol. 32, p. 29-37.

JOHNSON D. W. : JOHNSON R. T. \& TJOSVOLD D. (2000). "Constructive controversy: The value of intellectual opposition ". In M. Deutsch \& P. T. Coleman (éd.), The handbook of conflict resolution: theory and practice. San Francisco : Jossey-Bass, p. 65-85.

LICHT B. G. \& DWECK C. S. (1984). «Determinants of academic achievement: The interaction of children's achievement orientations with skill area ". Developmental Psychology, vol. 20, p. 628-636.
LOWRY N. \& JOHNSON D. W. (1981). « Effect of controversy on epistemic curiosity, achievement and attitudes ". The Journal of Social Psychology, vol. 115, p. 31-43.

MAEHR M. L. \& MIDGLEY C. (1991). « Enhancing students motivation : a schoolwide approach ". Educational Psychologist, vol. 26, p. 399-427.

MONTEIL J.-M. (1989). Éduquer et former: perspectives psycho-sociales. Grenoble : PUG.

Mugny G. ; Butera F. ; Quiamzade A. ; Dragulescu A. \& Tome A. (2003). "Comparaisons sociales des compétences et dynamiques d'influence sociale dans les tâches d'aptitudes ". L'Année psychologique, vol. 104, p. 469-496

Mugny G. ; De Paolis P. \& Carugati F. (1984). " Social regulations in cognitive development $"$. In W. Doise \& A. Palmonari (éd.), Social interaction in individual development. Cambridge: Cambridge University Press, p. 127-146.

Newman R. S. (1990). "Children's help-seeking in the classroom: The role of motivational factors and attitudes". Journal of Educational Psychology, vol. 82, p. 71-80.

Nicholls J. G. (1984). "Achievement motivation: Conceptions of ability, subjective experience, task choice, and performance ". Psychological Review, vol. 91, p. 328346.

Pérez J. A. ; Mugny G. ; Maggi J. ; Falomir J. M. \& Butera F. (1995). "L'élaboration du conflit. Les significations du conflit dans l'influence sociale ». Psychoscope, vol. 2, p. 11-14

Quiamzade A. \& Mugny G. (2001). "Social influence dynamics in aptitude tasks". Social Psychology of Education, vol. 4, n' $3-4$, p. 311-334.

SMITH K.; JOHNSON D. W. \& JOHnSON R. T. (1981). "Can conflict be constructive? Controversy versus concurrence seeking in learning groups ". Journal of Educational Psychology, vol. 73, p. 651-663. 